\title{
Evolución natural de la displasia septoóptica: análisis retrospectivo de 20 casos
}

\author{
Miguelina León-González, Juan J. García-Peñas, Diego Puertas-Bordallo, Miguel A. López-Pino, \\ Jesús Argente-Oliver, Verónica Cantarín-Extremera
}

Introducción. La displasia septoóptica (DSO) es la combinación variable de signos de disgenesia de línea media cerebral,
hipoplasia de nervios ópticos y disfunción hipotálamo-hipofisaria, asociándose, a veces, con un espectro variado de mal-
formaciones de la corteza cerebral. Objetivo. Describir la evolución natural y los hallazgos de neuroimagen en una serie de 20 pacientes diagnosticados.

Pacientes y métodos. Se revisan de forma retrospectiva las características epidemiológicas, clínicas y neurroradiológicas de 20 pacientes consecutivos diagnosticados de DSO entre enero de 1985 y enero de 2010. Se analizaron los datos de tomografía computarizada, resonancia magnética craneal, electroencefalograma, potenciales evocados visuales, valoración oftalmológica, cariotipo y estudio endocrinológico. En siete pacientes, se realizó estudio del gen Homeobox HESX1.

Resultados. El 60\% de los casos presentaba antecedentes patológicos en el primer trimestre de gestación, con las ecografías fetales normales. Clínicamente, destacaban manifestaciones visuales (85\%), alteraciones endocrinas (50\%), retraso mental (60\%) y crisis epilépticas (55\%). Un 55\% se asociaba a anomalías de migración neuronal. En un $45 \%$, la DSO era el único hallazgo de neuroimagen. Se realizó cariotipo a todos, siendo normal. El gen HESX1 fue positivo en dos de los siete casos estudiados (ambos con DSO aislada). Ninguno con mutación en el gen HESX1 presentaba consanguinidad familiar. No se realizó estudio genético a los padres.

Conclusiones. La DSO debe clasificarse como un síndrome malformativo heterogéneo, que asocia múltiples anomalías cerebrales, oculares, endocrinas y sistémicas. Las formas más graves se asocian con anomalías de la migración neuronal y de la organización cortical.

Palabras clave. Displasia septoóptica. Esquisencefalia. Hipoplasia del nervio óptico. Polimicrogiria. Resonancia magnética. Síndrome de Morsier.

\section{Introducción}

En 1956, De Morsier [1] describió un síndrome caracterizado por la ausencia o disgenesia del septo pelúcido (SP) asociada a hipoplasia de nervios ópticos, al que denominó displasia septoóptica (DSO). Desde entonces, los diversos estudios epidemiológicos, clínicos, anatómicos y de neuroimagen sugieren que la DSO representa una entidad anatomoclínica heterogénea que es la expresión final de diferentes anomalías prenatales, entre las que se incluyen daños isquémicos durante el primer o segundo trimestre de gestación (responsables de alteraciones de la migración neuronal y de la organización cortical) y deficiencias del mesénquima embrionario (causando anomalías de la línea media cerebral que se solapan con las características propias de la holoprosencefalia lobar) [2-7].

Los estudios que analizan series amplias de casos con DSO [8-11] describen esta entidad sindró- mica como una alteración disgenética de las estructuras de la línea media cerebral, incluyendo diversas combinaciones de hipoplasia o ausencia de SP (a veces, también del cuerpo calloso), hipoplasia/displasia de los nervios ópticos y disfunción hipotálamo-hipofisaria (con déficits hormonales que varían desde deficiencias hormonales aisladas al panhipopituitarismo). Este espectro de anomalías puede también asociarse con diversas alteraciones del parénquima cerebral, como esquisencefalias, polimicrogirias y displasias corticales focales [8-11].

Esta aparente heterogeneidad de la DSO contradice los hallazgos genéticos iniciales del grupo de Dattani [12-14], que sugerían que se trataba de un síndrome disgenético único por alteración en genes de la familia Homeobox. Esta confusión nosológica no se ha solucionado, a pesar de los resultados prometedores con el estudio de mutaciones en el gen HESX1 (un gen Homeobox que desempeña un importante papel en la prosencefalización y en el de-
Sección de Neurología (M LeónGonzález, J.J. García-Peñas, V. Cantarín-Extremera); Sección de Oftalmología (D. Puertas-Bordallo); Sección de Neurorradiología (M.A. López-Pino); Servicio de Endocrinología (J. Argente-Oliver); Hospital Infantil Universitario Niño Jesús; Madrid, España.

Correspondencia:

Dra. Miguelina León González. Sección de Neurología. Hospital Infantil Universitario Niño Jesús. Avda. Menéndez Pelayo, 65. E-28009 Madrid.

E-mail:

migueleongo@hotmail.com

Aceptado tras revisión externa: 01.02.12.

Cómo citar este artículo: León-González M, García-Peñas JJ, Puertas-Bordallo D, López-Pino MA, Argente-Oliver J, Cantarín-Extremera V. Evolución natural de la displasia septoóptica: análisis retrospectivo de 20 casos. Rev Neurol 2012; 54: 321-31.

c 2012 Revista de Neurología 
sarrollo de las estructuras de la línea media cerebral y la región hipotálamo-hipofisaria) [14].

Hoy en día, la etiología de una gran parte de los casos de DSO permanece aún sin definir. Este síndrome heterogéneo podría ser la expresión final de diversas anomalías genéticas (principalmente, genes reguladores de la prosencefalización basal) o bien de daños adquiridos intraútero (por isquemia o anoxia cerebral o por exposición a fármacos o tóxicos) [11].

Desde el punto de vista neurroradiológico, los pacientes con DSO han sido clasificados por Barkovich et al [2] en dos grupos anatómicos bien diferenciados en virtud de los hallazgos embriogénicos y neuropatológicos, incluyendo un subtipo con esquisencefalia o polimicrogiria, ventrículos laterales de tamaño normal, radiaciones ópticas normales y defectos incompletos del SP; y otros grupo de pacientes que asocian ausencia completa de SP e hipoplasia de la sustancia blanca periventricular (con desarrollo de ventriculomegalia), sin evidencia de anomalías de la migración o de la organización neuronal.

Este espectro malformativo de la DSO se puede clasificar, de acuerdo con la descripción anatomoclínica de Miller y Shevell [15], en una forma de DSO aislada (ausencia de SP, hipoplasia de nervios ópticos e hipoplasia de la sustancia blanca) y un grupo de DSO-plus (defectos parciales del SP asociados con diversas alteraciones de la migración o de la organización neuronal cortical).

El objetivo de esta publicación es describir la epidemiología, la evolución natural y los hallazgos de neuroimagen en una serie de 20 pacientes diagnosticados de DSO, analizando si existen diferencias anatomoclínicas y evolutivas entre los pacientes con DSO aislada y aquellos otros casos que se asocian con anomalías de la migración neuronal o alteraciones de la organización cortical cerebral, del tipo de la esquisencefalia, polimicrogiria o displasia cortical focal (síndrome DSO-plus).

\section{Pacientes y métodos}

Se revisaron las historias clínicas de los pacientes diagnosticados de DSO en el Hospital Universitario Niño Jesús de Madrid entre enero de 1985 y enero de 2010.

Tomando como criterios de DSO aquellas formas con alteración de estructuras de la línea media cerebral que asocien hipoplasia o ausencia de SP e hipoplasia o displasia de nervios ópticos y/o quiasma óptico definida por los estudios neuroanatómi- cos o por la repercusión funcional visual $[2,3,6,11]$, se han seleccionado 20 pacientes.

En todos los casos, se analizan: edad en el momento del diagnóstico, sexo, motivo de consulta, antecedentes familiares y personales, semiología neurológica, alteraciones visuales, anomalías endocrinológicas, malformaciones asociadas, hallazgos de neuroimagen, resultados de los estudios genéticos y evolución clínica.

En todos los pacientes, se analizaron también los datos de tomografía computarizada craneal, resonancia magnética (RM) cerebral, electroencefalograma, potenciales evocados visuales (PEV), valoración oftalmológica (agudeza visual, examen de motilidad ocular y oftalmoscopia), estudio endocrinológico (test de función hipotálamo-hipofisaria), cariotipo, valoración psicométrica -test de BrunetLèzine o escala de inteligencia de Wechsler para niños revisada (WISC-R), según la edad-y radiografías de esqueleto (serie ósea completa). En 10 casos, se hizo, además, una ecografía transfontanelar. En siete casos, se realizó estudio de mutaciones del gen Homeobox HESX1.

El seguimiento clínico de los pacientes osciló entre 1 y 18 años. En la actualidad, nueve pacientes superan los 18 años de edad.

\section{Resultados}

Las características de los 20 pacientes se reflejan en la tabla.

Sexo

De los 20 pacientes analizados, 14 eran varones (70\%), y 6 , mujeres (30\%).

\section{Edad en el momento del diagnóstico}

La edad en el momento del diagnóstico de la DSO osciló entre 1 mes y 13 años. Hasta un total de 16 pacientes (80\%) se diagnosticaron durante el primer año de vida.

\section{Antecedentes familiares}

En ningún paciente se encontró historia familiar de DSO ni de otras malformaciones del sistema nervioso central. Sólo en un caso se referían antecedentes familiares de crisis epilépticas (hermano mayor de uno de los pacientes, en el cual la RM cerebral fue normal). Uno de los pacientes tiene una hermana gemela univitelina normal. Tan sólo en dos 
casos existía consanguinidad en segundo grado en los progenitores (primos hermanos, de etnia gitana, en ambos casos).

\section{Antecedentes personales}

En 12 pacientes (60\%) existían antecedentes prenatales desfavorables, incluyendo: metrorragias en el primer trimestre $(n=8)$, prematuridad $(n=8)$, edad materna menor de 21 años $(n=5$ casos), diabetes gestacional $(n=4)$ y consumo de drogas (heroína y cocaína) durante la gestación $(n=4)$. Cuatro pacientes (20\%) presentaron puntuación en el test de Apgar menor de 6 a los 5 minutos. En 10 casos (50\%), el peso al nacer fue inferior a $2.500 \mathrm{~g}$.

\section{Motivo de consulta}

Los motivos de consulta incluyeron nistagmo ocular en ocho casos (40\%), crisis convulsivas en seis (30\%), déficit visual en tres (15\%) y retraso psicomotor (RPM) en otros tres (15\%).

\section{Alteraciones oftalmológicas}

Las anomalías de la vía visual estaban presentes en los 20 pacientes con DSO (100\%), incluyendo déficit de agudeza visual en 17 casos (85\%), nistagmo pendular en $14(70 \%)$, estrabismo convergente bilateral en 8 (40\%) y microftalmia en 5 (25\%). El fondo de ojo objetivó la presencia de hipoplasia del disco óptico (Fig. 1) en los 20 casos (100\%), de forma bilateral en 11 casos (55\%) y de forma unilateral o claramente asimétrica en 9 (45\%). Los PEV estaban alterados en 18 pacientes (90\%), con ausencia total de PEV en 6 casos (30\%) y aumento de latencias con disminución de amplitud en los otros 12 (70\%), siendo la conducción claramente asimétrica en 8 casos (40\%).

\section{Síntomas y signos neurológicos}

Diecisiete casos (85\%) presentaron anomalías neurológicas durante su seguimiento, incluyendo 12 pacientes $(60 \%)$ con RPM, siendo éste grave en 3 casos, moderado en 5 y leve en los 4 restantes. Tres pacientes (15\%) desarrollaron signos de trastorno del espectro autista. Las crisis epilépticas estaban presentes en 11 casos (55\%): 8 como crisis parciales y 3 como crisis generalizadas. Se objetivaron déficits motores en 11 pacientes (55\%): 6s como parálisis cerebral infantil tipo diaplejía espástica, 3 como hemiplejía y 2 como tetraparesia espástica. Cinco casos (25\%) desarrollaron microcefalia evolutiva y 3
(15\%) presentaron macrocefalia progresiva. El electroencefalograma fue patológico en 11 casos (55\%), mostrando anomalías focales en 8 casos (40\%) y descargas generalizadas de punta-onda en los 3 restantes (15\%).

La valoración psicométrica (test de Brunet-Lèzine) o la WISC-R en los pacientes con DSO aislada mostraron un retraso psicomotor leve, y en los pacientes con DSO-plus, un retraso moderado-grave.

\section{Anomalías endocrinológicas}

Estaban presentes en 10 pacientes (50\%), incluyendo talla por debajo del percentil p3 en 7 casos (35\%), criptorquidia bilateral con micropene en 5 (25\%), hipoglucemia recurrente en 5 (25\%) y diabetes insípida central en $4(20 \%)$. Existía panhipopituitarismo en 5 casos (25\%), déficit aislado de la hormona del crecimiento $(\mathrm{GH})$ en 4 (20\%) y déficit aislado de la hormona estimulante del tiroides (TSH) en 1 (5\%).

\section{Malformaciones asociadas}

Se objetivaron en 6 casos (30\%), incluyendo luxación congénita bilateral de cadera en 4 y pies zambos congénitos en 2 pacientes.

\section{Fenotipo dismórfico}

Hasta 11 pacientes (55\%) presentaban diversos grados de dismorfia facial, incluyendo prominencia frontal, hipertelorismo, raíz nasal ancha y deprimida y philtrum labial largo.

\section{Estudios de neuroimagen (Figs. 1 a 5)}

En 9 casos (45\%), la DSO fue el único hallazgo neurorradiológico. En los 11 pacientes restantes (55\%), la DSO se asoció a anomalías de migración neuronal y organización cortical (DSO-plus), incluyendo: 7 esquisencefalias (bilateral en 5 casos y unilateral en 2) y 4 polimicrogirias perisilvianas (unilateral en 1 caso y bilateral en los otros 3). Se objetivó hipoplasia de las vías ópticas (quiasma o nervios ópticos) en 11 casos (55\%). Por otra parte, se apreció la existencia de hipoplasia de estructuras hipotálamohipofisarias en 6 casos, anomalías del tallo hipofisario en 3 pacientes y ectopia de la neurohipófisis en otros 3 .

\section{Resultados de los estudios genéticos}

A todos los pacientes se les había realizado un cariotipo que había sido normal. Se investigaron mu- 
Tabla. Características de los 20 pacientes con displasia septoóptica (DSO).

\begin{tabular}{|c|c|c|c|c|c|c|c|c|c|c|c|c|c|}
\hline & Sexo & Edad & $\mathrm{AF}$ & AP & MC & Oftalmol. & SNC & Endocr. & Sistémico & NeuroRx & EEG & PEV & Evolución \\
\hline 1 & V & 1 mes & Consang. & $\begin{array}{c}\text { Metrorragias } \\
\text { SFA } \\
\text { Edad }<21 \text { años }\end{array}$ & Nistagmo & $\begin{array}{c}\text { Nistagmo } \\
\text { Déficit AV } \\
\text { HNO-BL } \\
\text { Microftalmia }\end{array}$ & $\begin{array}{l}\text { Macrocefalia } \\
\text { TEA }\end{array}$ & $\begin{array}{c}\text { Panhipopituitarismo } \\
\text { Criptorquidia } \\
\text { bilateral } \\
\text { Hipoglucemia }\end{array}$ & $\begin{array}{l}\text { Luxación } \\
\text { caderas } \\
\text { Dismorfia }\end{array}$ & DSO aislada & $\mathrm{N}$ & Patológicos & $\begin{array}{c}\text { Ambliopía } \\
\text { grave }\end{array}$ \\
\hline 2 & V & 3 meses & - & $\begin{array}{c}\text { Metrorragias } \\
\text { SFA } \\
\text { Drogas } \\
\text { PRN }<2.500 \mathrm{~g}\end{array}$ & Crisis & $\begin{array}{l}\text { Nistagmo } \\
\text { Déficit AV } \\
\text { HNO-UL }\end{array}$ & $\begin{array}{c}\text { RPM } \\
\text { Crisis } \\
\text { PCl } \\
\text { Microcefalia }\end{array}$ & - & - & $\begin{array}{c}\text { DSO con } \\
\text { esquisencefalia }\end{array}$ & Gen. & Patológicos & \\
\hline 3 & V & 4 meses & - & $\begin{array}{c}\text { Metrorragias } \\
\text { Drogas } \\
\text { PRN }<2.500 \mathrm{~g}\end{array}$ & RPM & $\begin{array}{l}\text { Nistagmo } \\
\text { Déficit AV } \\
\text { HNO-UL }\end{array}$ & $\begin{array}{c}\text { RPM } \\
\text { Crisis } \\
\text { PCI } \\
\text { Microcefalia }\end{array}$ & - & - & $\begin{array}{c}\text { DSO con } \\
\text { esquisencefalia }\end{array}$ & Gen. & Patológicos & \\
\hline 4 & V & 6 meses & - & $\begin{array}{c}\text { Diabetes } \\
\text { Pretérmino } \\
\text { PRN }<2.500 \mathrm{~g} \\
\text { Edad }<21 \text { años }\end{array}$ & Nistagmo & $\begin{array}{l}\text { Nistagmo } \\
\text { Déficit AV } \\
\text { Estrabismo } \\
\text { Microftalmia } \\
\text { HNO-BL }\end{array}$ & RPM & $\begin{array}{c}\text { DI } \\
\text { GH } \\
\text { Hipoglucemia } \\
\text { Talla baja }\end{array}$ & $\begin{array}{l}\text { Luxación } \\
\text { caderas } \\
\text { Dismorfia }\end{array}$ & DSO aislada & $\mathrm{N}$ & Patológicos & $\begin{array}{c}\text { Ambliopía } \\
\text { grave }\end{array}$ \\
\hline 5 & V & 7 meses & - & $\begin{array}{c}\text { Diabetes } \\
\text { Pretérmino } \\
\text { PRN }<2.500 \mathrm{~g}\end{array}$ & Nistagmo & $\begin{array}{l}\text { Nistagmo } \\
\text { Déficit AV } \\
\text { Estrabismo } \\
\text { HNO-BL }\end{array}$ & $\begin{array}{l}\text { RPM } \\
\text { Crisis } \\
\text { PCl }\end{array}$ & - & - & $\begin{array}{c}\text { DSO con } \\
\text { esquisencefalia }\end{array}$ & Gen. & Patológicos & \\
\hline 6 & V & 4 meses & - & $\begin{array}{l}\text { Metrorragias } \\
\text { Pretérmino } \\
\text { PRN }<2.500 \mathrm{~g} \\
\text { Edad }<21 \text { años }\end{array}$ & $\begin{array}{l}\text { Deficiencia } \\
\text { visual }\end{array}$ & $\begin{array}{c}\text { Nistagmo } \\
\text { Déficit AV } \\
\text { Microftalmia } \\
\text { HNO-BL }\end{array}$ & $\begin{array}{c}\text { Macrocefalia } \\
\text { TEA }\end{array}$ & $\begin{array}{c}\text { DI } \\
\text { CH } \\
\text { Hipoglucemia } \\
\text { Talla baja }\end{array}$ & Dismorfia & DSO aislada & $\mathrm{N}$ & Patológicos & $\begin{array}{c}\text { Ambliopía } \\
\text { grave }\end{array}$ \\
\hline 7 & V & 5 meses & - & $\begin{array}{l}\text { Metrorragias } \\
\text { Pretérmino } \\
\text { PRN }<2.500 \mathrm{~g} \\
\text { Edad }<21 \text { años }\end{array}$ & $\begin{array}{l}\text { Deficiencia } \\
\text { visual }\end{array}$ & $\begin{array}{c}\text { Nistagmo } \\
\text { Déficit AV } \\
\text { Microftalmia } \\
\text { HNO-BL }\end{array}$ & $\begin{array}{c}\text { Macrocefalia } \\
\text { TEA }\end{array}$ & $\begin{array}{c}\text { DI } \\
\text { TSH }\end{array}$ & $\begin{array}{l}\text { Luxación } \\
\text { caderas } \\
\text { Dismorfia }\end{array}$ & DSO aislada & $\mathrm{N}$ & Patológicos & $\begin{array}{l}\text { Ambliopía } \\
\text { grave }\end{array}$ \\
\hline 8 & V & 7 meses & - & $\begin{array}{c}\text { Diabetes } \\
\text { Pretérmino } \\
\text { PRN }<2.500 \mathrm{~g} \\
\text { Edad }<21 \text { años }\end{array}$ & $\begin{array}{l}\text { Deficiencia } \\
\text { visual }\end{array}$ & $\begin{array}{l}\text { Nistagmo } \\
\text { Déficit AV } \\
\text { Estrabismo } \\
\text { Microftalmia } \\
\text { HNO-BL }\end{array}$ & $\begin{array}{l}\text { RPM } \\
\text { Crisis } \\
\text { PCl }\end{array}$ & - & - & $\begin{array}{c}\text { DSO con } \\
\text { polimicrogiria }\end{array}$ & Focal & Patológicos & $\begin{array}{c}\text { Ambliopía } \\
\text { grave }\end{array}$ \\
\hline 9 & V & 8 meses & - & $\begin{array}{c}\text { Drogas } \\
\text { Pretérmino } \\
\text { PRN }<2.500 \mathrm{~g}\end{array}$ & RPM & $\begin{array}{l}\text { Nistagmo } \\
\text { Déficit AV } \\
\text { Estrabismo } \\
\text { HNO-UL }\end{array}$ & $\begin{array}{c}\text { RPM } \\
\text { Crisis } \\
\text { PCl } \\
\text { Microcefalia }\end{array}$ & - & - & $\begin{array}{c}\text { DSO con } \\
\text { esquisencefalia }\end{array}$ & Focal & Patológicos & \\
\hline 10 & V & 5 meses & - & - & Nistagmo & $\begin{array}{l}\text { Nistagmo } \\
\text { Déficit AV } \\
\text { HNO-BL }\end{array}$ & Macrocefalia & $\begin{array}{c}\text { Panhipopituitarismo } \\
\text { Criptorquidia } \\
\text { bilateral } \\
\text { Talla baja }\end{array}$ & $\begin{array}{l}\text { Luxación } \\
\text { caderas } \\
\text { Dismorfia }\end{array}$ & DSO aislada & $\mathrm{N}$ & Patológicos & $\begin{array}{c}\text { Ambliopía } \\
\text { grave }\end{array}$ \\
\hline 11 & V & 3 meses & - & $\begin{array}{c}\text { Drogas } \\
\text { Metrorragias } \\
\text { Pretérmino } \\
\text { PRN }<2.500 \mathrm{~g}\end{array}$ & Crisis & HNO-UL & $\begin{array}{l}\text { RPM } \\
\text { Crisis } \\
\text { PCl }\end{array}$ & - & - & $\begin{array}{c}\text { DSO con } \\
\text { polimicrogiria }\end{array}$ & Focal & Patológicos & \\
\hline 12 & M & 5 meses & - & - & Nistagmo & $\begin{array}{l}\text { Nistagmo } \\
\text { Déficit AV } \\
\text { HNO-BL }\end{array}$ & Macrocefalia & $\begin{array}{c}\text { DI } \\
\text { GH } \\
\text { Hipoglucemia } \\
\text { Talla baja }\end{array}$ & $\begin{array}{c}\text { Pies zambos } \\
\text { Dismorfia }\end{array}$ & DSO aislada & $\mathrm{N}$ & Patológicos & \\
\hline
\end{tabular}


Tabla. Características de los 20 pacientes con displasia septoóptica (DSO) (cont.).

\begin{tabular}{|c|c|c|c|c|c|c|c|c|c|c|c|c|c|}
\hline & Sexo & Edad & $\mathrm{AF}$ & AP & MC & Oftalmol. & SNC & Endocr. & Sistémico & NeuroRx & EEG & PEV & Evolución \\
\hline 13 & M & $\begin{array}{c}11 \\
\text { meses }\end{array}$ & - & - & Nistagmo & $\begin{array}{l}\text { Nistagmo } \\
\text { Déficit AV } \\
\text { Estrabismo } \\
\text { HNO-BL }\end{array}$ & - & $\begin{array}{c}\text { Panhipopituitarismo } \\
\text { Criptorquidia } \\
\text { bilateral }\end{array}$ & Dismorfia & DSO aislada & $\mathrm{N}$ & Patológicos & \\
\hline 14 & M & 9 meses & - & $\begin{array}{c}\text { Metrorragias } \\
\text { SFA } \\
\text { Pretérmino } \\
\text { PRN }<2.500 \mathrm{~g}\end{array}$ & Crisis & HNO-UL & $\begin{array}{l}\text { RPM } \\
\text { Crisis } \\
\mathrm{PCl}\end{array}$ & - & - & $\begin{array}{c}\text { DSO con } \\
\text { polimicrogiria }\end{array}$ & Focal & Normales & $\begin{array}{l}\text { Epilepsia } \\
\text { refractaria }\end{array}$ \\
\hline 15 & M & 5 meses & - & - & Nistagmo & $\begin{array}{l}\text { Nistagmo } \\
\text { Déficit AV } \\
\text { HNO-BL }\end{array}$ & - & $\begin{array}{c}\text { Panhipopituitarismo } \\
\text { Criptorquidia } \\
\text { bilateral } \\
\text { Talla baja }\end{array}$ & Dismorfia & DSO aislada & $\mathrm{N}$ & Patológicos & $\begin{array}{l}\text { Ambliopía } \\
\text { grave }\end{array}$ \\
\hline 16 & $M$ & 6 meses & - & - & Nistagmo & $\begin{array}{l}\text { Nistagmo } \\
\text { Déficit AV } \\
\text { Estrabismo } \\
\text { HNO-BL }\end{array}$ & - & $\begin{array}{c}\text { Panhipopituitarismo } \\
\text { Talla baja }\end{array}$ & Dismorfia & DSO aislada & $\mathrm{N}$ & Patológicos & $\begin{array}{l}\text { Ambliopía } \\
\text { grave }\end{array}$ \\
\hline 17 & M & 13 años & $\begin{array}{l}\text { Hermano } \\
\text { con } \\
\text { epilepsia } \\
\text { consang. }\end{array}$ & - & Crisis & $\begin{array}{l}\text { Déficit AV } \\
\text { Estrabismo } \\
\text { HNO-UL }\end{array}$ & $\begin{array}{l}\text { RPM } \\
\text { Crisis } \\
\mathrm{PCl}\end{array}$ & $\begin{array}{c}\mathrm{GH} \\
\text { Criptorquidia bilateral } \\
\text { Hipoglucemia } \\
\text { Talla baja }\end{array}$ & $\begin{array}{l}\text { Dismorfia } \\
\text { Pies zambos }\end{array}$ & $\begin{array}{c}\text { DSO con } \\
\text { polimicrogiria }\end{array}$ & Focal & Patológicos & $\begin{array}{l}\text { Epilepsia } \\
\text { refractaria }\end{array}$ \\
\hline 18 & V & 6 años & - & - & Crisis & $\begin{array}{c}\text { Déficit AV } \\
\text { Estrabismo } \\
\text { HNO-UL }\end{array}$ & $\begin{array}{c}\text { RPM } \\
\text { Crisis } \\
\mathrm{PCl}\end{array}$ & - & Dismorfia & $\begin{array}{c}\text { DSO con } \\
\text { esquisencefalia }\end{array}$ & Focal & Patológicos & $\begin{array}{l}\text { Epilepsia } \\
\text { refractaria }\end{array}$ \\
\hline 19 & V & 2 años & - & - & RPM & $\begin{array}{l}\text { Déficit AV } \\
\text { HNO-UL }\end{array}$ & $\begin{array}{l}\text { RPM } \\
\text { Crisis } \\
\mathrm{PCl}\end{array}$ & - & - & $\begin{array}{c}\text { DSO con } \\
\text { esquisencefalia }\end{array}$ & Focal & Patológicos & $\begin{array}{c}\text { Epilepsia } \\
\text { refractaria }\end{array}$ \\
\hline 20 & V & 2 años & - & $\begin{array}{c}\text { Diabetes } \\
\text { Metrorragias } \\
\text { SFA }\end{array}$ & Crisis & HNO-UL & $\begin{array}{c}\text { RPM } \\
\text { Crisis } \\
\mathrm{PCl}\end{array}$ & - & - & $\begin{array}{c}\text { DSO con } \\
\text { esquisencefalia }\end{array}$ & Focal & Normales & $\begin{array}{l}\text { Epilepsia } \\
\text { refractaria }\end{array}$ \\
\hline
\end{tabular}

AF: antecedentes familiares; AP: antecedentes personales; AV: agudeza visual; BL: bilateral; DI: diabetes insípida; EEC: electroencefalograma; Endocr.: hallazgos endocrinológicos; Gen.: trazado electroencefalográfico generalizado; GH: hormona del crecimiento; HNO: hipoplasia del nervio óptico; MC: motivo de consulta; M: mujer; N: normal; NeuroRx: hallazgos de neuroimagen; Oftalmol.: hallazgos oftalmológicos; PCl: parálisis cerebral infantil; PRN: peso del recién nacido; RPM: retraso psicomotor; SFA: sufrimiento fetal agudo; SNC: hallazgos neurológicos; TEA: trastorno del espectro autista; TSH: hormona estimulante del tiroides; UL: unilateral; V: varón.

taciones en el gen HESX1/Hesx 1 en 7 de los 20 pacientes con DSO (35\%), incluyendo 5 casos con DSO aislada y 2 con DSO-plus. Las mutaciones heterocigotas en el gen Homeobox HESX1 se encontraron tan sólo en 2 de los 7 casos investigados $(28,5 \%)$, perteneciendo ambos al grupo de DSO aislada (el $40 \%$ de este subgrupo). Ambas mutaciones eran de tipo missense o aberrantes, codificando un cambio localizado de aminoácidos en la secuenciación del gen $H E S X 1$. Ninguno de los pacientes con mutación en el gen HESX1 presentaba consanguinidad en los familiares. No se realizó estudio genético a los padres.

\section{Evolución clínica}

Un total de 8 pacientes (40\%) han evolucionado hacia déficits visuales grave y 5 (25\%) han desarrollado una epilepsia refractaria.

\section{Discusión}

La ausencia de SP se ha referido en relación con diversas situaciones, incluyendo malformaciones del sistema nervioso central (DSO, holoprosencefalia, agenesia de cuerpo calloso, secuencia de Chiari tipo II 
Figura 1. Oftalmoscopia con hipoplasia de papila óptica (imagen de 'doble contorno').

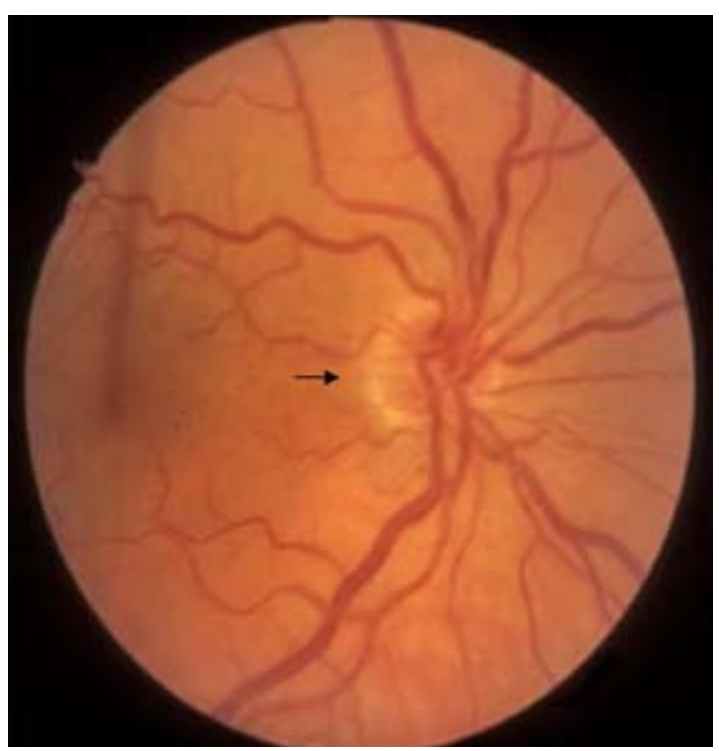

y encefaloceles), hidrocefalias obstructivas, procesos encefaloclásticos (hidranencefalia, porencefalia) y ausencia aislada del SP $[2,5,7,10,11,15,16]$.

\section{Patología}

Se han publicado pocos trabajos que revisen las anomalías neuropatológicas que se encuentran en la DSO [17-19]. Existe habitualmente ausencia de SP, pero también se han referido casos con hipoplasia o adelgazamiento de éste $[17,18]$. Los nervios ópticos suelen ser hipoplásicos, pero también pueden ser normales [17]. En el globo ocular, se aprecia menor número de células ganglionares retinianas $\mathrm{y}$, por lo tanto, de sus axones, existiendo de esta forma un menor número de fibras nerviosas en el nervio óptico, pero con sus cubiertas inalteradas $[6,17]$. En algunos casos, se han descrito, además, displasias retinianas [6]. Los nervios olfatorios pueden ser hipoplásicos, normales o ausentes [17]. También se han descrito ausencia de núcleos hipotalámicos [17], ausencia-hipoplasia del lóbulo posterior de la hipófisis [6,17-19], anomalías de los cuerpos geniculados laterales [17], agenesia de la porción anterior del cuerpo calloso [2,17-19], agenesia del vermis cerebeloso $[17,18]$ y rombencefalosinapsis [19]. Los hemisferios cerebrales pueden ser normales o bien mostrar anomalías de la migración neuronal o de la
Figura 2. Corte coronal de resonancia magnética cerebral que muestra ausencia del septo pelúcido e hipoplasia de nervios ópticos.

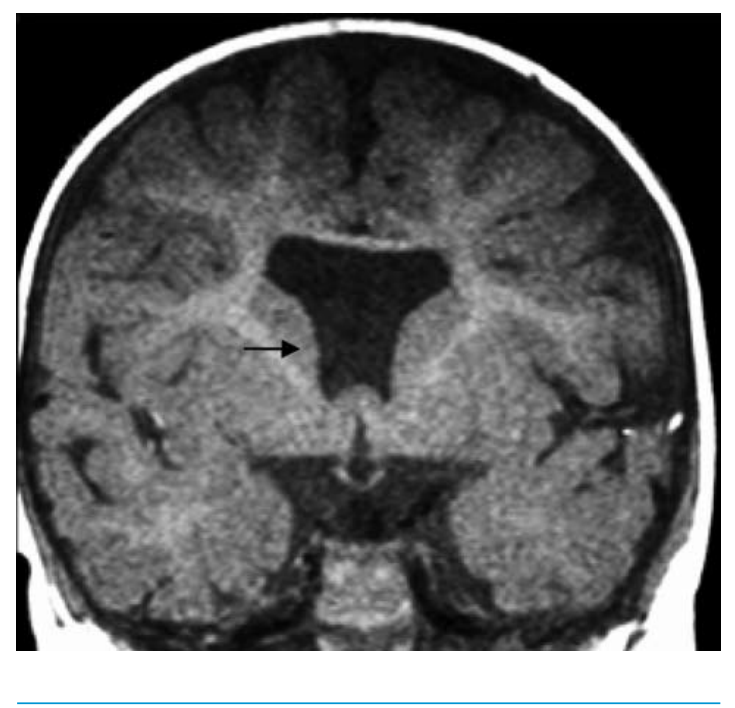

organización cortical, incluyendo esquisencefalia, porencefalia, polimicrogiria, heterotopías neuronales y displasias corticales [2-20].

Hasta un $30 \%$ de los casos de nuestra serie presentaba anomalías de las estructuras hipotálamohipofisarias, lo que confirma el alto porcentaje de anomalías cerebrales asociadas a la DSO.

\section{Etiología}

La etiología de la DSO sigue siendo desconocida en la gran mayoría de los casos. En la literatura se recogen básicamente tres teorías etiopatogénicas. La primera de ellas considera que la afectación de los nervios ópticos y de las estructuras cerebrales son coincidentes en el tiempo, debido a una disgenesia cerebral anterior de probable origen vascular (esencialmente, por alteraciones en el territorio tributario de la arteria cerebral anterior) y que afectaría al embrión entre la sexta y la octava semana de la embriogénesis $[21,22]$. Para algunos autores, esta alteración del neurodesarrollo supondría una forma parcial de holoprosencefalia [6]. Por otra parte, la segunda teoría defiende la existencia de una degeneración de las fibras ópticas, bien formadas previamente, de forma secundaria a una lesión del sistema nervioso central de diversa naturaleza [22]. La tercera teoría sugiere una base genética. Hay formas autosómicas recesivas; sin embargo, tan sólo se ha descrito una familia con más de un miembro 
Figura 3. Corte axial de resonancia magnética craneal con ausencia del septo pelúcido (flecha negra) y esquisencefalia bilateral de labio cerrado (flechas blancas).

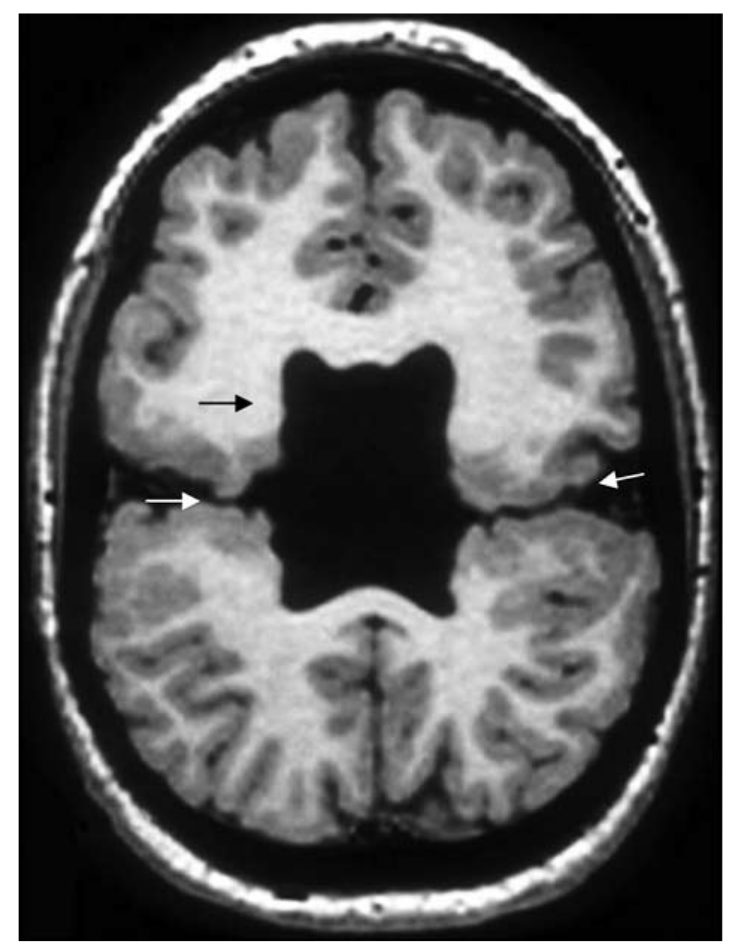

afecto [23]. La hipótesis genética apunta a posibles defectos localizados en el gen HESX1/hesx1 (gen Homeobox fundamental para el desarrollo de la prosencefalización) localizado en el brazo corto del cromosoma 3 [12-14]. Dado que mutaciones en HESX1 sólo se han encontrado en un $10 \%$ de las DSO, parece que podrían existir otros genes, es decir, que la DSO mostraría heterogeneidad genética $[6,12-14,24]$. Por otra parte, se ha especulado con el papel de otros genes que participan en el desarrollo de la línea media cerebral y de las estructuras oculares, incluyendo, sobre todo, al gen SRY/SOX2 (sex determining region Y-box 2) [11].

En nuestra serie, no hemos encontrado casos con agregación familiar. Tan sólo destaca la presencia de dos familias con consanguinidad en segundo grado (primos hermanos) de forma similar a lo descrito en otras revisiones [6]. Sin embargo, en la población que analizamos, encontramos mutaciones heterocigotas de novo en el gen HESX1 en un 28,5\% de los casos testados. Esta mayor proporción de alteraciones genéticas puede deberse a que no estu-
Figura 4. Reconstrucción tridimensional de imagen de resonancia magnética cerebral, corte axial, con displasia septoóptica asociada a esquisencefalia bilateral de labio cerrado.

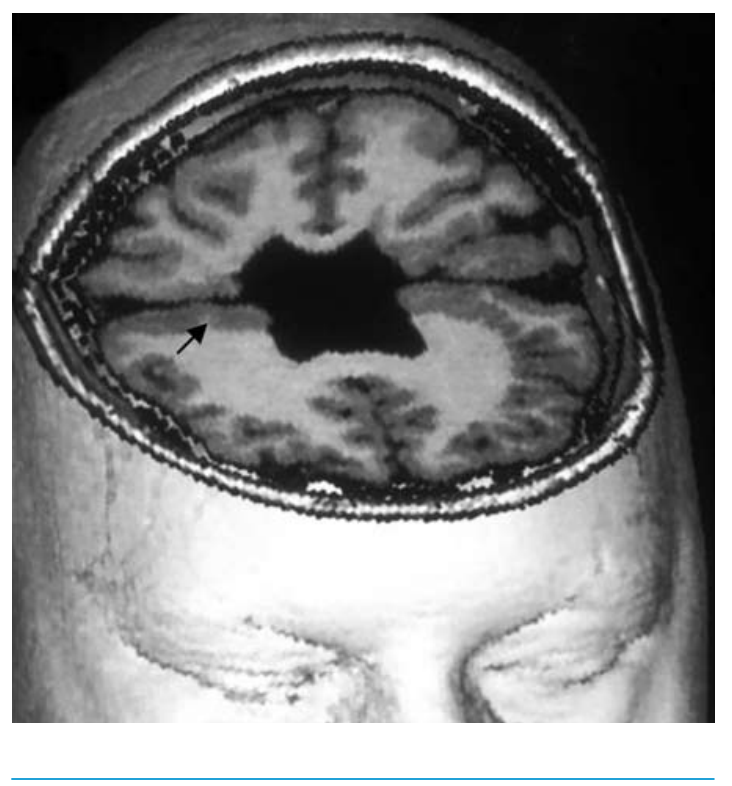

diamos a toda la población de DSO y a que la práctica totalidad de los pacientes a los que se realizó el estudio genético correspondía a la forma de DSO aislada, donde es mucho más probable la hipótesis de una teoría genética con afectación aislada de genes Homeobox.

Se han descrito diversas asociaciones de DSO con factores prenatales desfavorables, incluyendo diabetes gestacional $[11,25]$, exposición materna a fármacos y drogas (fenciclidina, fenitoína, quinina, cocaína, heroína) $[11,26]$, edad de las gestantes por debajo de 21 años [11,25-27], infecciones congénitas por citomegalovirus, metrorragias durante el primer trimestre de gestación y prematuridad o bajo peso al nacer [22]. En nuestro grupo poblacional, es remarcable la presencia de antecedentes prenatales patológicos hasta en un $60 \%$ de los casos, destacando la presencia de metrorragias en el primer trimestre de gestación (40\%), la prematuridad (40\%) y el bajo peso al nacer (50\%).

Con respecto al tipo de DSO, las formas con DSO aislada presentaron antecedentes prenatales desfavorables solamente en un $44 \%$, y las formas con DSO-plus lo referían hasta en un $72 \%$. Todos los casos con exposición a drogas intraútero (cocaína o heroína) se correspondieron con formas tipo DSO-plus. 
Figura 5. Corte axial de resonancia magnética cerebral ponderado en $\mathrm{T}_{2}$ que muestra hipoplasia de nervios, quiasma y cintillas ópticas.

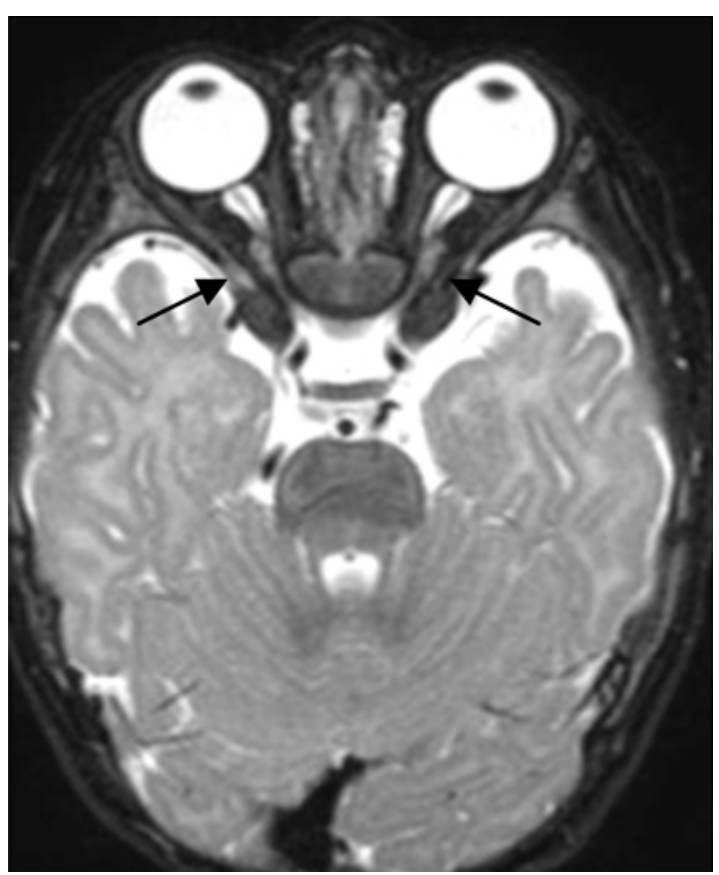

\section{Datos poblacionales}

Sexo

Aunque en otras series $[6,11,22]$ no se han recogido diferencias significativas en cuanto a la predilección por uno u otro sexo, destaca en nuestro grupo poblacional el predominio del sexo masculino (70\%).

\section{Edad}

Aunque el diagnóstico de estos pacientes se difiere a veces hasta la edad escolar [6], destaca en nuestra serie que hasta a un $80 \%$ de los niños se les diagnosticó antes de los 12 meses de edad. Los casos con un diagnóstico más tardío (4 pacientes entre 2 y 13 años) presentaban clínica neurológica predominante en el contexto de una DSO-plus con anomalías de la migración neuronal o de la organización cortical.

\section{Presentación clínica}

La presentación clínica es variable, oscilando desde formas con encefalopatías graves a casos con anomalías visuales o endocrinológicas aisladas. Los pa- cientes consultaban habitualmente por síntomas de disfunción oftalmológica o neurológica $[6,22]$.

En nuestra serie, el motivo más frecuente de consulta fue el nistagmo (40\%), seguido de las crisis convulsivas (30\%). Con respecto al tipo de DSO, destaca que las formas con DSO aislada consultaron exclusivamente por nistagmo o déficit visual, y que las formas con DSO-plus lo hicieron principalmente por crisis convulsivas o evidencia de RPM.

\section{Anomalías oculares}

Las manifestaciones oftalmológicas son frecuentes y casi constantes en la DSO, incluyendo [1-6,22,28]: disminución de la agudeza visual (casi siempre evidente durante el primer año de vida), defectos campimétricos, como hemianopsias bitemporales (si se afecta el quiasma óptico), defectos sectoriales o centrocecales, alteración de la visión espacial, nistagmo uni o bilateral (rotatorio o pendular), alteraciones foveolares, estrabismos (convergentes o divergentes), defectos en los reflejos pupilares, microftalmía, blefarofimosis, obstrucciones de la vía lagrimal, aniridia y colobomas. Existen, sin embargo, casos con visión normal [29]. Es característica la presencia de papilas ópticas hipoplásicas en el fondo de ojo, muchas veces con la imagen típica de 'micropapilas con doble anillo o doble contorno'. La presencia de hipoplasia óptica es decisiva para el diagnóstico de DSO y suele ser bilateral, aunque puede ser asimétrica o claramente unilateral [1-6, 22]. Varios estudios demuestran que no hay asociación fiable entre el aspecto anatómico (estudios de dimensiones del disco óptico por ecografía) y la función visual del nervio [30]. Los electrorretinogramas son, habitualmente, normales, pero los PEV están frecuentemente alterados, de forma uni o bilateral, dependiendo del estado de las aferencias de la vía óptica [1-6,22,28].

En nuestra serie, todos los pacientes tenían anomalías de la vía óptica y hasta un $85 \%$ de los niños presentaba algún síntoma o signo de disfunción oftalmológica, predominando el déficit de agudeza visual (85\%) y el nistagmo ocular (70\%). Con respecto al tipo de DSO, destaca que todos los pacientes con DSO aislada presentaban semiología de disfunción visual, y que esta situación sólo se encontraba en un 73\% de las formas con DSO-plus.

La hipoplasia de papilas ópticas fue un hallazgo constante en todos nuestros pacientes con DSO (Fig. 1), siendo ésta bilateral en todos los casos con DSO aislada y predominando la afectación unilateral o asimétrica en las formas con DSO plus (el 82\% de los casos). 
Los PEV estaban alterados en el 90\% de nuestros casos con DSO, encontrándose los dos casos normales (el 10\% del total) en el contexto de las DSOplus. Por otra parte, en el grupo de DSO-plus, las anomalías de los PEV eran unilaterales o bien claramente asimétricas hasta en el 73\% de los casos.

\section{Anomalías endocrinológicas}

Hasta dos tercios de los pacientes pueden presentar signos de disfunción hipotalámico-hipofisaria, ya sea como déficits hormonales aislados o como panhipopituitarismo $[6,11,17,22,31-33]$, destacando, principalmente, la talla baja en relación con déficit de la GH hasta en un 70\% de los casos [6,22,31-33]. También se han descrito otras alteraciones [34,35], como diabetes insípida central por déficit de la hormona antidiurética (el 25\% de los casos), hipotiroidismo por déficit de TSH (20\%), hipoglucemias recurrentes por déficit de $\mathrm{GH}$, hipocortisolismo, hipogonadismo y alteraciones puberales, como pubertad precoz. Estas alteraciones hormonales provocan insuficiencias en los correspondientes órganos diana y trastornos multisistémicos, incluyendo daños cerebrales graves [33-35].

En nuestra serie, las anomalías endocrinológicas se presentaron en un 50\% del total de las DSO, destacando la presencia de talla baja evolutiva en un $35 \%$ de los casos y la presencia de un panhipopituitarismo evidente hasta en el $25 \%$ de los niños. Con respecto al tipo de DSO, destaca que todos los pacientes con DSO aislada presentaban déficit endocrinológico, y que esta situación sólo se encontraba en un único caso (9\%) con DSO-plus.

Es importante recalcar que existe riesgo de daño cerebral permanente o muerte en los pacientes con DSO y afectación del eje hipotálamo-hipofisiario, por lo cual resulta fundamental el diagnóstico precoz de posibles deficiencias hormonales y el consiguiente tratamiento hormonal sustitutivo [35]. En concreto, el hipocortisolismo se asocia con riesgo de muerte súbita en niños y adultos, principalmente durante viremias y crisis febriles que precipitan una crisis suprarrenal.

La deshidratación y las alteraciones de la termorregulación asociadas con la diabetes insípida central pueden empeorar todavía más la situación clínica [35].

Son necesarias para ello mediciones hormonales seriadas en sangre y orina, y se debe conocer, además, que estas anomalías de la función endocrina pueden producirse tardíamente, por lo cual, en estos pacientes, las revisiones endocrinológicas resultan obligatorias.

\section{Anomalías neurológicas}

Los síntomas y signos neurológicos son inconstantes en los casos con DSO aislada. Así, las crisis epilépticas ocurren en una minoría de pacientes y no es frecuente el retraso mental ni los déficits focales $[3,6,22,36]$. Sin embargo, en el grupo de pacientes con DSO asociada a anomalías corticales cerebrales (DSO-plus), son frecuentes las convulsiones (casi siempre como epilepsia parcial), los déficits motores (con distintas formas evolutivas de parálisis cerebral infantil), la microcefalia adquirida, los trastornos de aprendizaje y el RPM [3,6,7,11,22,29]. Por otra parte, se han descrito también casos con autismo y DSO [6,37]. Algunas de estas manifestaciones pueden ser secundarias tanto a las malformaciones neurológicas como a trastornos metabólicos producidos por el hipopituitarismo [35].

Un $85 \%$ de los pacientes presentó anomalías neurológicas evolutivas, destacando la presencia de RPM (60\%) y epilepsia (55\%). Con respecto al tipo de DSO, un $66 \%$ de los pacientes con DSO aislada presentaba clínica neurológica (predominando los casos con macrocefalia o semiología autista), mientras que en el grupo con DSO-plus, la afectación neurológica se objetivaba en todos los pacientes (predominando el RPM, la epilepsia, la microcefalia y los déficits motores tipo parálisis cerebral infantil).

\section{Hallazgos de neuroimagen}

Los estudios de neuroimagen muestran hipoplasia o ausencia completa del SP, resultando la característica apariencia de astas frontales cuadrangulares $[2,6,11,15,22,37]$. En apenas un 50\% de los casos [2, $3,6,11,22,30]$, los estudios de RM cerebral permiten identificar claramente la hipoplasia de nervios ópticos o quiasma. Es más frecuente, sin embargo, objetivar la hipoplasia de vías ópticas o del hipotálamo en virtud de signos indirectos de imagen, como dilatación bullosa del receso anterior del tercer ventrículo y aumento de la cisterna periquiasmática [3, 29]. Otras anomalías de neuroimagen asociadas con la DSO incluyen agenesia de cuerpo calloso, hipoplasia hipotálamo-hipofisaria, ectopia hipofisaria, anomalías del tallo hipofisario, porencefalia, esquisencefalia, polimicrogiria, heterotopías periventriculares, displasia cortical focal e hidrocefalia $[2,3,5$, 6,11,15,16,22,36-38].

La ecografía cerebral es una prueba de utilidad muy limitada en la valoración de la DSO $[2,15,37,38]$ $\mathrm{y}$, habitualmente, tan sólo permite constatar la ausencia del SP, que, a veces, puede confundirse con una variante de la normalidad tipo quiste del SP. 
La tomografía computarizada cerebral confirma la posible ausencia de DSO, pero no suele ser útil para definir las anomalías asociadas de la migración neuronal o de la organización cortical $[2,15,37,38]$.

Por todo ello, se debe considerar que la RM cerebral (Figs. 2 a 5) es la prueba de elección para delimitar el espectro malformativo de una posible DSO $[2,3,6,15,37,38]$.

La RM craneal fue esencial en el diagnóstico de los pacientes de nuestra serie, no sólo para describir hipoplasia de las vías ópticas, sino también para identificar anomalías de la migración neuronal (hasta en 11 de ellos).

\section{Tipos anatomoclínicos de DSO}

En virtud de los hallazgos en la RM cerebral, Barkovich $[2,38]$ propone clasificar las DSO en dos grupos bien diferenciados:

- Grupo de DSO aisladas, sin anomalías de migración neuronal. Presentan ausencia del SP e hipoplasia de la sustancia blanca periventricular con ventriculomegalia asociada. En algunos casos, se objetiva, además, una hipoplasia de la porción anterior de la hoz del cerebro y de la rodilla del cuerpo calloso. Se piensa que este grupo representa una variante leve de la holoprosencefalia lobar. Clínicamente, predominan los signos de disfunción hipotalámico-hipofisaria (principalmente, déficit de la GH o panhipopituitarismo) y las anomalías oculares bilaterales y simétricas, siendo infrecuentes las anomalías neurológicas tipo RPM o crisis epilépticas. En nuestra serie, este tipo de anomalía representa un $45 \%$ del total de los casos.

- Grupo de DSO asociadas a anomalías de la migración neuronal o de la organización cortical (DSOplus). Suele tratarse de esquisencefalias, polimicrogirias y heterotopías de la sustancia gris. Existe, habitualmente, una hipoplasia o una ausencia parcial del SP, aunque se han descrito casos con ausencia completa del SP. La sustancia blanca de las radiaciones ópticas es normal. Son frecuentes los signos neurológicos graves (epilepsia, RPM o parálisis cerebral infantil). Las anomalías visuales no son constantes y la hipoplasia óptica suele ser unilateral; y, cuando es bilateral, es claramente asimétrica (en relación con el área afecta por la polimicrogiria o la esquisencefalia). Las alteraciones endocrinológicas son raras en este grupo y no se encuentran, habitualmente, en un primer plano. En nuestra serie, este tipo de anomalía representa un 55\% del total de los casos, asociándose la DSO con esquisencefalia o polimicrogiria.
Existen, por otra parte, casos de DSO asociada a ectopia hipofisaria posterior, que podrían constituir un tercer subgrupo independiente $[2,3,38]$.

En conclusión, la DSO es un síndrome malformativo de las estructuras de la línea media cerebral con una expresión clínica y neurorradiológica variable y muy heterogénea. En nuestra serie, las formas más graves pertenecen al grupo de DSO-plus asociada a anomalías de la migración neuronal o de la organización cortical cerebral.

Se debe sospechar la presencia de un síndrome de DSO ante la evidencia de hipoplasia del nervio óptico o del quiasma óptico asociada a alteraciones estructurales de la línea media (sobre todo ausencia o hipogenesia del SP y disfunción hipotálamo-hipofisaria.

La presencia de anomalías en los genes Homeobox es un hallazgo inconstante, que habitualmente aparece como mutaciones de novo, por lo cual el consejo genético en estas familias debe orientarse como formas de bajo riesgo de recurrencia.

La colaboración interdisciplinar (oftalmología, neurología, pediatría y endocrinología) es básica tanto en el diagnóstico como en el seguimiento de estos pacientes.

Bibliografía

1. De Morsier G. Études sur les dysgraphies cranioencéphaliques. Agénésie du septum lucidum avec malformation du tractus optique. La dysplasie septo-optique. Schw Arch Neurol Psych 1956; 77: 267-92

2. Barkovich AJ, Fram EK, Norman D. Septo-optic dysplasia: MR imaging. Radiology 1989; 171: 189-92.

3. Brodsky MC, Glasier CM. Optic nerve hypoplasia: clinical significance of associated central nervous system abnormalities on magnetic resonance imaging. Arch Ophthalmol 1993; 111: 66-74.

4. Ouvrier R, Billson F. Optic nerve hypoplasia: a review. J Child Neurol 1986; 1: 181-8.

5. Leech RW, Shuman RM. Holoprosencephaly and related midline cerebral anomalies: a review. J Child Neurol 1986; 1: 3-18.

6. Polizzi A, Pavone P, Iannetti P, Manfré L, Ruggieri M. Septo-optic dysplasia complex: a heterogeneous malformation syndrome. Pediatr Neurol 2006; 34: 66-71.

7. Gasparetto EL, Warszawiak D, De Carvalho-Neto A Benites-Filho PR, Bruck I, Antoniuk S. Displasia septo-óptica plus: relato de caso. Arq Neuro-Psiquiatr 2003; 61: 671-6.

8. Campbell CL. Septo-optic dysplasia: a literature review. Optometry 2003; 74: 417-26.

9. Borchert M, Garcia-Filion P. The syndrome of optic nerve hypoplasia. Curr Neurol Neurosci Rep 2008; 8: 395-403.

10. Volpe P, Campobasso G, De Robertis V, Rembouskos G. Disorders of prosencephalic development. Prenat Diagn 2009; 29: 340-54.

11. Webb EA, Dattani MT. Septo-optic dysplasia. Eur J Med Genet 2010; 18: 393-7.

12. Dattani MT, Martínez-Barberá JP, Thomas PQ, Brickman JM, Gupta R, Wales JK, et al. HESX1: a novel gene implicated in a familial form of septo-optic dysplasia. Acta Paediatr Suppl 1999; 88: 49-54. 
13. Dattani ML, Martínez-Barberá J, Thomas PQ, Brickman JM, Gupta R, Wales JK, et al. Molecular genetics of septo-optic dysplasia. Horm Res 2000; 53 (Suppl 1): S26-33.

14. Dattani MT, Robinson IC. HESX1 and septo-optic dysplasia. Rev Endocr Metab Disord 2002; 3: 289-300.

15. Miller SP, Shevell MI. Septo-optic dysplasia plus: a spectrum of malformations of cortical development. Neurology 2000; 54: 1701-3.

16. Siejka S, Strefling AM, Urich $H$. Absence of septum pellucidum and polymicrogyria: a forme fruste of the porencephalic syndrome. Clin Neuropathol 1989; 8: 174-8.

17. Roessmann U, Velasco ME, Small EJ, Hori A. Neuropathology of 'septo-optic dysplasia' (De Morsier syndrome) with immunohistochemical studies of the hypothalamus and pituitary gland. J Neuropathol Exp Neurol 1987; 46: 597-608.

18. Roessmann U. Septo-optic dysplasia (SOD) or De Morsier syndrome. J Clin Neuroophthalmol 1989; 9: 156-9.

19. Hayashi M, Sakamoto K, Kurata K, Nagata J, Satoh J, Morimatsu Y. Septo-optic dysplasia with cerebellar hypoplasia in Cornelia de Lange syndrome. Acta Neuropathol (Berlin) 1996; 92: 625-30.

20. Pascual-Castroviejo I, Viaños J. Esquisencefalia frente a porencefalia. Rev Neurol 2011; 52: 623-4.

21. Lubinski MS. Hypothesis: septo-optic dysplasia is a vascular disruption sequence. Am J Med Genet 1997; 69: 235-6.

22. Ramos-Fernández JM, Martínez-San Millán J, Barrio R, Yturriaga R, Lorenzo G, Aparicio JM. Displasia septo-óptica: presentación de 6 pacientes estudiados mediante RM cerebral y discusión de su patogenia. An Esp Pediatr 1996; 45: 614-8.

23. Benner JD, Preslan MW, Gratz E, Joslyn J, Schwartz M, Kelman S. Septo-optic dysplasia in two siblings. Am J Ophthalmol 1990; 109: 632-7.

24. Thomas PQ, Dattani MT, Brickman JM, McNay D, Warne G, Zacharin $\mathrm{M}$, et al. Heterozygous HESX1 mutations associated with isolated congenital pituitary hypoplasia and septo-optic dysplasia. Hum Mol Genet 2001; 10: 39-45.

25. Donat JF. Septo-optic dysplasia in an infant of a diabetic mother. Arch Neurol 1981; 38: 590-1.
26. Orrico A, Galli L. Septo-optic dysplasia with digital anomalies associated with maternal multidrug abuse during pregnancy. Eur J Neurol 2002; 9: 679-82.

27. Michaud J, Mizrahi EM, Urich H. Agenesis of the vermis with fusion of the cerebellar hemispheres, septo-optic dysplasia and associated anomalies. Report of a case. Acta Neuropathol (Berlin) 1982; 56: 161-6.

28. Brooks DB, Subramanian PS. Monocular temporal hemianopsia with septo-optic dysplasia. J Neuroophthalmol 2006; 26: 195-6.

29. Skarf B, Hoyt CS. Optic nerve hypoplasia in children. Association with anomalies of the endocrine and CNS. Arch Ophthalmol 1984; 102: 62-7.

30. Acers TE. Optic nerve hypoplasia: septo-optic-pituitary dysplasia syndrome. Trans Am Ophthalmol Soc 1981; 79: 425-57.

31. Izenberg N, Rosenblum M, Parks JS. The endocrine spectrum of septo-optic dysplasia. Clin Pediatr 1984; 23: 632-6.

32. Arslanian SA, Rothfus WE, Foley TP, Becker DJ. Hormonal, metabolic, and neuroradiologic abnormalities associated with septo-optic dysplasia. Acta Endocrinol 1984; 107: 282-8.

33. Margalith D, Tze WJ, Jan JE. Congenital optic nerve hypoplasia with hypothalamic-pituitary dysplasia. A review of 16 cases. Am J Dis Child 1985; 139: 361-6.

34. Stangel M, Vogeley KT, Jandeck C, Boegner F, Marx P, Koch HC. Septo-optic dysplasia (De Morsier syndrome). Nervenarzt 1998; 69: 352-6.

35. Brodsky MC, Conte FA. Sudden death in septo-optic dysplasia. Report of 5 cases. Arch Ophthalmol 1997; 115: 66-70.

36. Morishima A, Aranoff GS. Syndrome of septo-optic-pituitary dysplasia: the clinical spectrum. Brain Dev 1986; 8: 233-9.

37. Polizzi A, Pavone P, Iannetti P, Gambardella A, Ruggieri M. CNS findings in three cases of septo-optic dysplasia, including one with semilobar holoprosencephaly. Am J Med Genet 2005; 136: 357-60.

38. Barkovich AJ. Pediatric neuroimaging. 3 ed. Philadelphia: Lippincott Williams \& Wilkins; 2000

\section{Natural course of septo-optic dysplasia: retrospective analysis of $\mathbf{2 0}$ cases}

Introduction. Septo-optic dysplasia (SOD) is the variable combination of signs of dysgenesis of the midline of the brain, hypoplasia of the optic nerves and hypothalamus-pituitary dysfunction, which is sometimes associated with a varied spectrum of malformations of the cerebral cortex.

Aims. To describe the natural history and neuroimaging findings in a series of 20 diagnosed patients.

Patients and methods. We review the epidemiological, clinical and neuroimaging characteristics of 20 consecutive patients diagnosed with SOD between January 1985 and January 2010. Data obtained from computerised tomography, magnetic resonance imaging of the head, electroencephalogram, visual evoked potentials, ophthalmological evaluation, karyotyping and endocrinological studies were analysed. In seven patients, a study of the gene Homeobox HESX1 was conducted.

Results. Pathological antecedents in the first three months of gestation were presented by $60 \%$ of the cases, with normal results in the foetal ultrasound scans. Clinically, the most striking features were visual manifestations (85\%), endocrine disorders (50\%), mental retardation (60\%) and epileptic seizures (55\%). Fifty-five per cent were associated to abnormal neuronal migration. In 45\%, SOD was the only finding in the neuroimaging scans. Karyotyping was performed in all cases, the results being normal. Gene HESX1 was positive in two of the seven cases studied (both with isolated SOD). None of those with mutation in gene HESX1 presented familial consanguinity. No gene study was conducted with the parents.

Conclusions. SOD must be classified as a heterogeneous malformation syndrome, which is associated to multiple brain, ocular, endocrine and systemic anomalies. The most severe forms are associated with abnormal neuronal migration and cortical organisation.

Key words. Magnetic resonance imaging. Morsier syndrome. Optic nerve hypoplasia. Polymicrogyria. Schizencephaly. Septooptic dysplasia. 